\title{
Characterization of Three LYSO Crystal Batches
}

\author{
Fan Yang, Rihua Mao, Liyuan Zhang and Ren-Yuan Zhu \\ 256-48, HEP, California Institute of Technology, Pasadena, CA 91125, USA \\ E-mail: zhu@ @ep.caltech.edu
}

\begin{abstract}
We report on three LYSO crystal batches characterized at the Caltech crystal laboratory for future HEP experiments: Twenty-five $20 \mathrm{~cm}$ long crystals for the SuperB experiment; twelve $13 \mathrm{~cm}$ long crystals for the Mu2e experiment and $62314 \times 14 \times 1.5 \mathrm{~mm}$ plates with five holes for a $\mathrm{LYSO} / \mathrm{W}$ Shashlik matrix for a beam test at Fermilab. Optical and scintillation properties measured are longitudinal Transmittance, light output and FWHM energy resolution. Correlations between these properties are also investigated.
\end{abstract}

Key Words: LYSO crystal; Scintillation property; Correlation

\section{Introduction}

Because of their high density $\left(7.4 \mathrm{~g} / \mathrm{cm}^{3}\right)$, short radiation length $(1.14 \mathrm{~cm})$, fast $(40 \mathrm{~ns})$ and bright (4 times $\mathrm{BGO})$ scintillation, cerium doped lutetium oxyorthosilicate $\left(\mathrm{Lu}_{2} \mathrm{SiO}_{5}: \mathrm{Ce}, \mathrm{LSO}\right)$ [1-5] and lutetium yttrium oxyorthosilicate $\left(\mathrm{Lu}_{2(1-x)} \mathrm{Y}_{2 \mathrm{x}} \mathrm{SiO}_{5}\right.$ :Ce, LYSO) [6-8] crystals have attracted a broad interest in the high energy physics community pursuing precision electromagnetic calorimeter for future high energy physics experiments [9-13]. Their excellent radiation hardness against gamma-rays $[14,15]$, neutrons [16] and charged hadrons [17] also makes them a preferred material for calorimeters to be operated in a severe radiation environment, such as the HL-LHC.

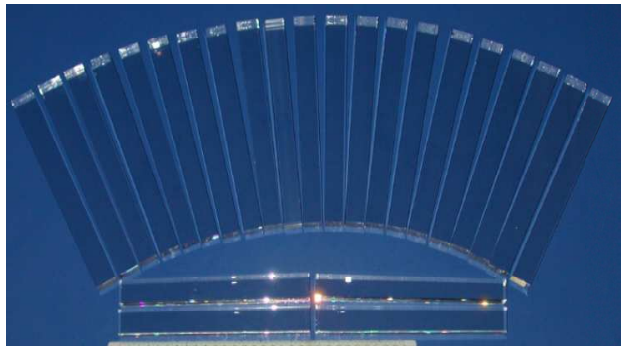

Figure 1. Twenty five $20 \mathrm{~cm}$ long LYSO crystals of tapered shape for SuperB

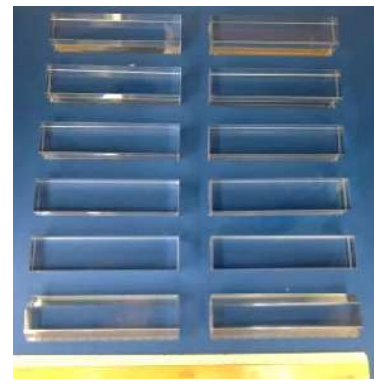

Figure 2. Twelve $13 \mathrm{~cm}$ long LYSO crystals for $\mathrm{Mu} 2 \mathrm{e}$

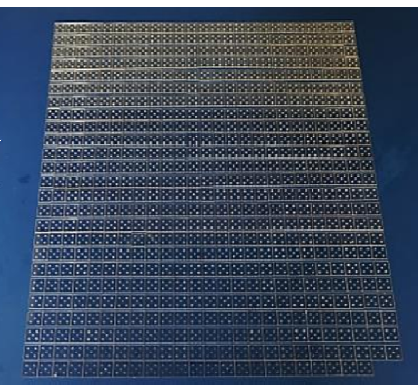

Figure 3. $62314 \times 14$ x 1.5 $\mathrm{mm}$ LYSO plates for a LYSO/W Shashlik matrix 
All crystal vendors are encouraged to provide crystals for future HEP experiments. The choice of vendors will be made by each experiment based upon cost and performance. It is the hope that the paper is useful for crystal vendors to improve the quality of their crystals. Figure 1 shows twenty five $20 \mathrm{~cm}$ long LYSO crystals of tapered geometry with about $20 \times 20 \mathrm{~mm}^{2}$ at the small end and $23 \times 23$ $\mathrm{mm}^{2}$ at the large end for the SuperB experiment. They were grown by Czochralski method at SaintGobain (SG) Corporation, Shanghai Institute of Ceramics (SIC) and Sichuan Institute of Piezoelectric and Acousto-optic Technology (SIPAT). Figure 2 shows ten rectangular Mu2e crystals with dimension of $30 \times 30 \times 130 \mathrm{~mm}^{3}$ and other two hexagonal crystals of $18.6 \mathrm{~mm}$ wide and $130 \mathrm{~mm}$ long. All the Mu2e crystals were grown at SIC. Figure 3 shows $62314 \times 14 \times 1.5 \mathrm{~mm}^{3}$ LYSO plates grown at SIC for a LYSO/W Shashlik matrix [11]. There are five holes in these plates to allow four wavelength shifters and one monitoring fiber to go through. All six faces of these crystals are polished.

The longitudinal transmittance (LT) was measured using a PerkinElmer LAMBDA 950 UV/Vis spectrophotometer with double beam, double monochromator and a large crystal compartment equipped with an integrating sphere. The systematic uncertainty in repeated measurements is $0.15 \%$. The scintillation light output (LO) of SuperB crystals was measured by using a pair of Hamamatsu S8664-55 APDs because the SuperB project chose APD as the photo-detector. Their FWHM energy resolution (ER) was measured by a Hamamatsu R1306 PMT with a bi-alkali photo-cathode and a borosilicate glass window because the noise contribution APD readout is not adequate for the ER measurement in laboratory with radioactive sources. The scintillation LO and ER were also measured by the Hamamatsu R1306 PMT for Mu2e crystals and Shashlik plates. In the LO measurement for long crystals the large (tapered crystals) or one (rectangular and hexagonal crystals) end of the crystals was coupled to the PMT with Dow Corning 200 fluid, while all other faces of the crystal were wrapped with Tyvek paper. $\mathrm{A}{ }^{22} \mathrm{Na}$ source was used to excite the crystals, and provided the coincidence trigger. In the LO measurement for LYSO plates, one large face was coupled to the PMT with an air gap, while all other faces were wrapped with Tyvek paper. $\mathrm{A}{ }^{137} \mathrm{Cs}$ source was used to excite the crystals. The $\gamma$-ray peak positions were obtained by a simple Gaussian fit. The systematic 
uncertainty in repeated measurements is less than $1 \%$. Systematic uncertainties of our measurement were defined as RMS/mean of ten measurements for the same sample.

\section{Properties of Long LYSO Crystals for the SuperB and Mu2e Experiments}

\subsection{Longitudinal Transmittance and Emission Weighted Longitudinal Transmittance}

Figure 4 shows the LT at $420 \mathrm{~nm}$ for the SuperB LYSO crystals with divergences of less than $2 \%$ for crystals produced by each vendor, where $420 \mathrm{~nm}$ was chosen since the emission peak of LYSO crystals excited by gamma-rays is at $420 \mathrm{~nm}$ [18]. The LO of LYSO crystals is related to the LT at 420 $\mathrm{nm}$. Therefore the LT at $420 \mathrm{~nm}$ is essential to the crystal quality control. Among three venders SIPAT crystals have better average LT at $420 \mathrm{~nm}$. Figure 5 shows the LT at $420 \mathrm{~nm}$ for the Mu2e LYSO crystals. Rectangular and hexagonal crystals have consistent LT with an overall divergence of less than $1 \%$, where two crystals of different shape cut from the same ingot are shaded with red color. All crystals have LT at $420 \mathrm{~nm}$ better than the $75 \%$ specification, indicating good optical quality.

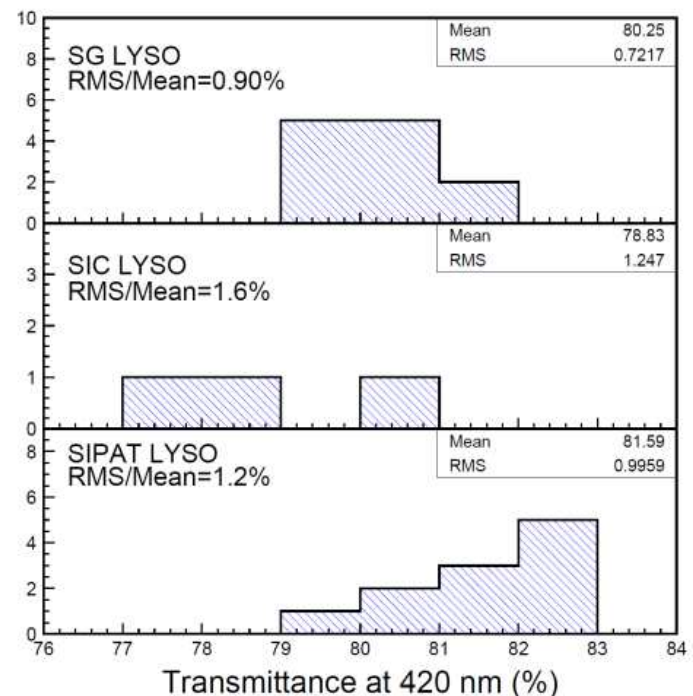

Figure 4. LT at $420 \mathrm{~nm}$ measured for twety five SuperB LYSO crystals

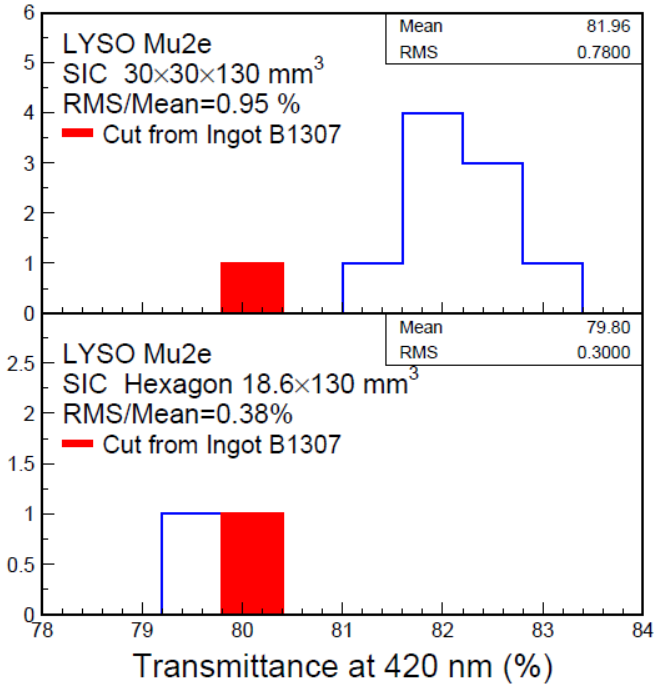

Figure 5. LT at $420 \mathrm{~nm}$ measured for twelve Mu2e LYSO crystals

Figures 6 and 7 show emission weighted longitudinal transmittance (EWLT) for SuperB and Mu2e crystals respectively, where EWLT is defined as [9]: 
EWLT presents crystal transmittance in the entire emission spectrum so is a better representation for crystal's transparency than the transmittance at $420 \mathrm{~nm}$. Figure 6 shows that crystals grown at SaintGobain and SIPAT have better EWLT than that from SIC. Figure 7 shows that the rectangular and hexagon crystals have consistent EWLT, where two crystals cut from the same ingot are shaded with red color. A larger divergence for EWLT than the LT at $420 \mathrm{~nm}$ was observed since EWLT is affected by both transmittance and the UV cutoff wavelength, which is affected by the cerium concentration in the crystal [6]. A small divergence at a level of $1.7 \%$ was observed for the Saint-Gobain crystals as well as the Mu2e SIC crystals, indicating consistent cerium doping in these batches.

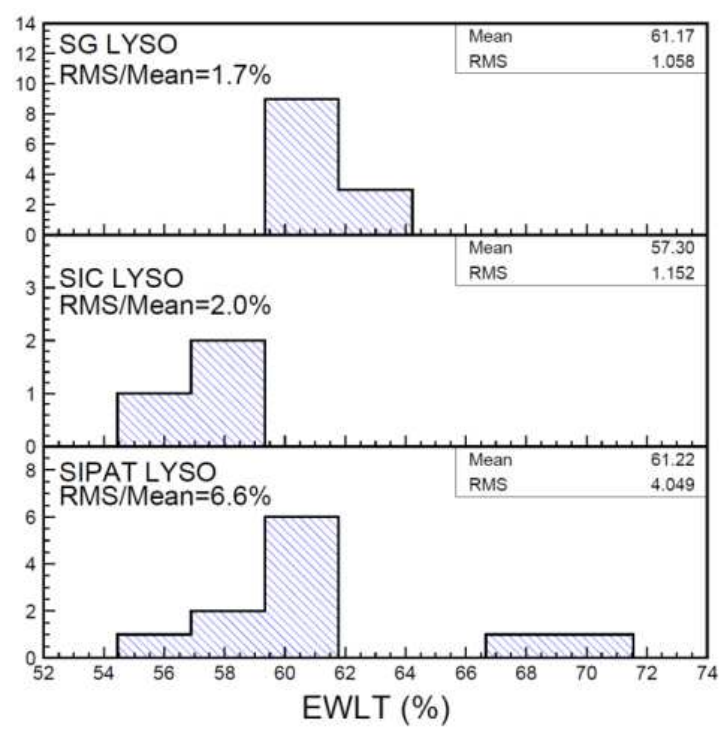

Figure 6. EWLT measured for twenty five SuperB LYSO crystals

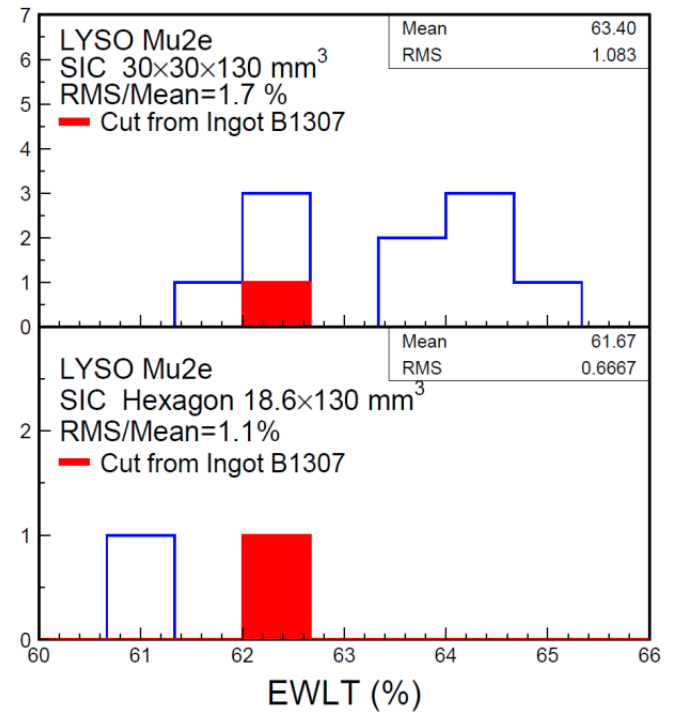

Figure 7. EWLT measured for twelve Mu2e LYSO crystals

\subsection{Light Output and FWHM Energy Resolution}

Figure 8 shows the LO measured by a pair of Hamamatsu S8664-55 APDs for twenty five SuperB LYSO crystals. While the SIPAT crystals have the best LO among three vendors, the Saint-Gobain crystals have the best consistency at about 3\%. Figure 9 shows the LO measured by a Hamamatsu 

appear to have higher LO than hexagonal ones cut from the same ingot. Further investigation, including a detailed ray-tracing simulation, however, is needed to understand this difference.

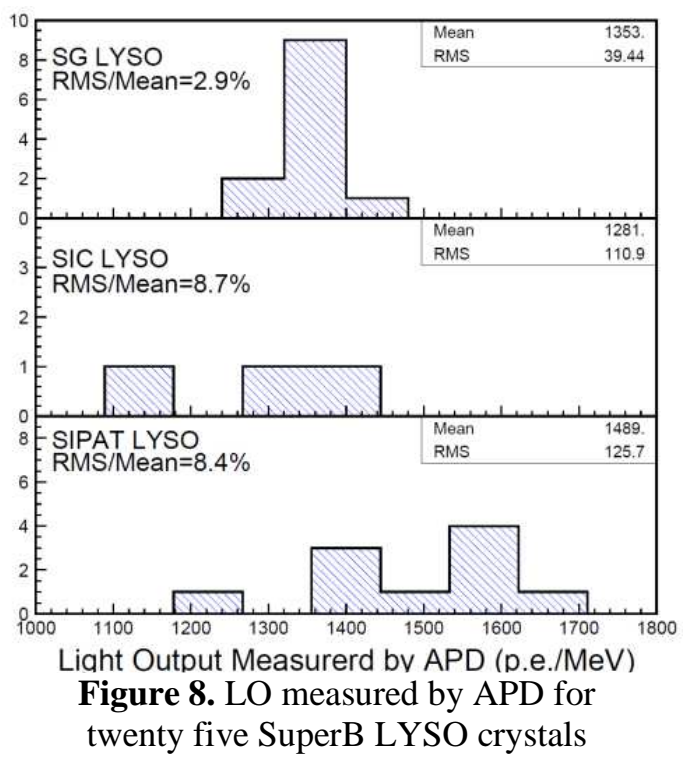

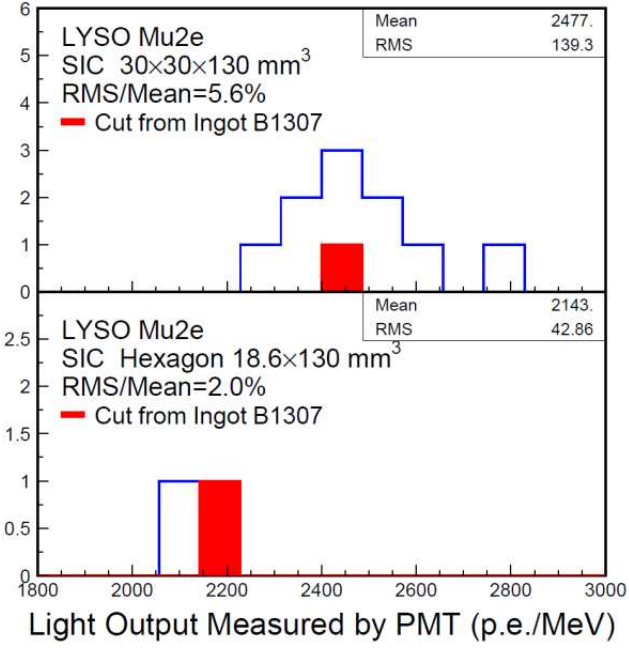

Figure 9. LO measured by PMT for twelve Mu2e LYSO crystals

Figure 10 shows the FWHM ER measured by a Hamamatsu R1306 PMT for twenty five SuperB crystals excited by $511 \mathrm{keV}$ gamma rays. The Saint-Gobain crystals have the best ER with a divergence of less than 2\%. Figure 11 shows the FWHM ER measured by the Hamamatsu R1306 PMT for twelve Mu2e crystals excited by $511 \mathrm{keV}$ gamma rays. Consistent ER between the rectangular and hexagonal crystals is observed. The ER divergence of SuperB LYSO is less than that of LO. It is due to the different readout device in the ER and LO measurements. The ER of SuperB LYSO was measured by a Hamamatsu R1306 PMT and LO was measured by a pair of Hamamatsu S8664-55 APDs. The noise of APD is larger than that of PMT. Because of the noise contribution, APD readout shows higher ER divergence of SuperB LYSO. The ER and LO of Mu2e LYSO were measured by a Hamamatsu R1306 PMT and the consistent divergence was observed in rectangular Mu2e LYSO. 


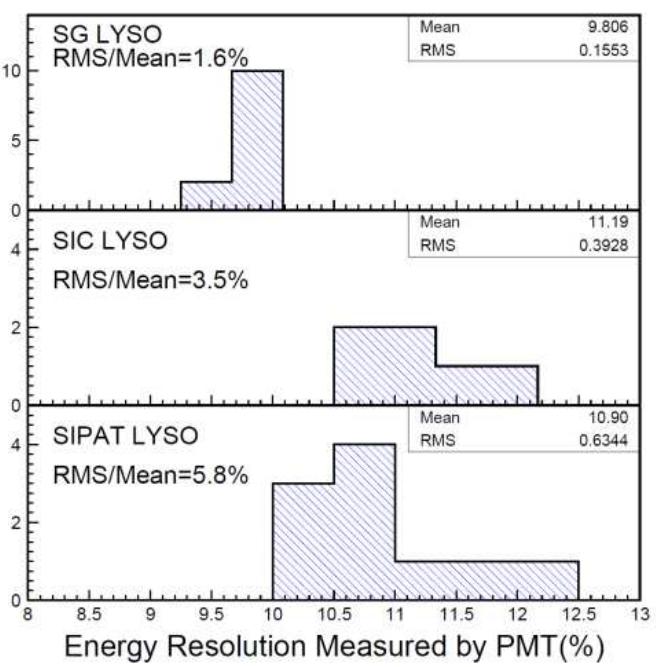

Figure 10. ER measured by PMT for twenty five SuperB LYSO crystals

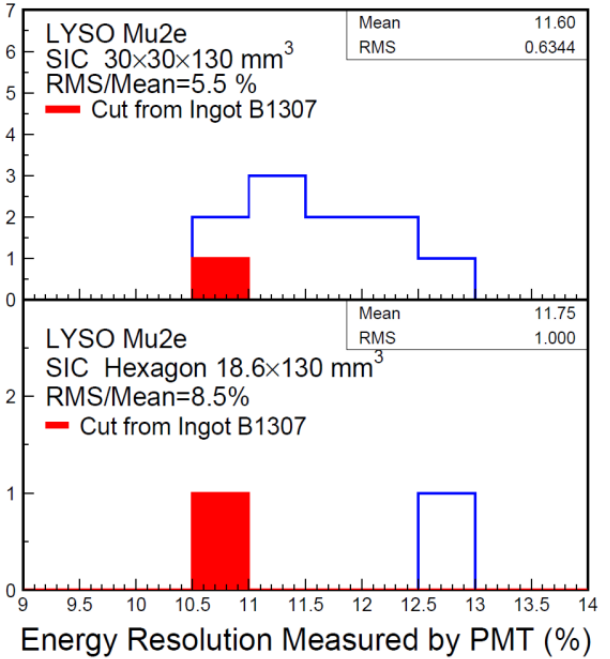

Figure 11. ER measured by PMT for twelve Mu2e LYSO crystals

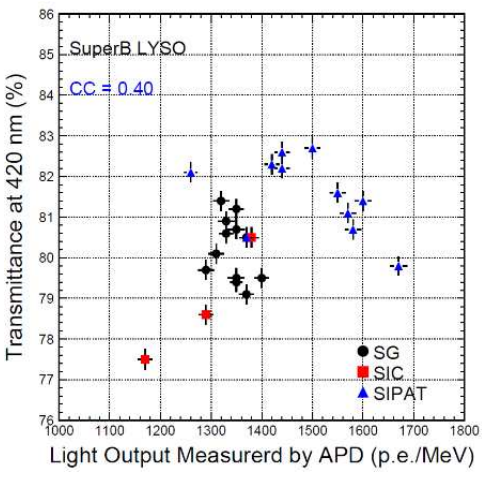

Figure 12. Correlation between the LO and the LT at $420 \mathrm{~nm}$ of 25 SuperB crystals

$$
\mathrm{CC}=\frac{\sum(x-\bar{x})(y-\bar{y})}{\sqrt{\sum(x-\bar{x})^{2} \sum(y-\bar{y})^{2}}}
$$

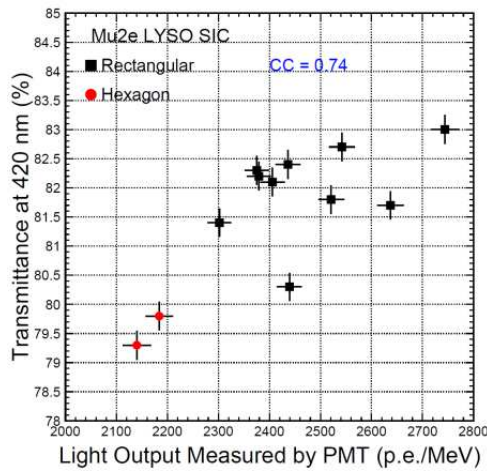

Figure 13. Correlation between the LO and the LT at $420 \mathrm{~nm}$ of $12 \mathrm{Mu} 2 \mathrm{e}$ crystals

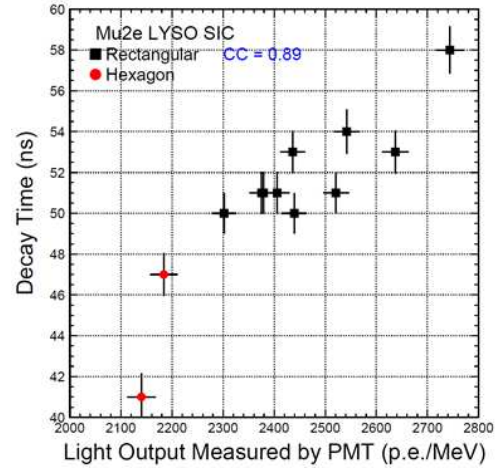

Figure 14. Correlation between the LO and the Decay time of $12 \mathrm{Mu} 2 \mathrm{e}$ crystals
105

Figure 13 shows a higher positive correlation coefficient at $74 \%$ observed between the $\mathrm{LO}$ and the LT at $420 \mathrm{~nm}$ for twelve Mu2e crystals than $40 \%$ observed for the SuperB crystals because of 
108

109

110

111

112

113

114

115

116

117

118

119

120

121

122

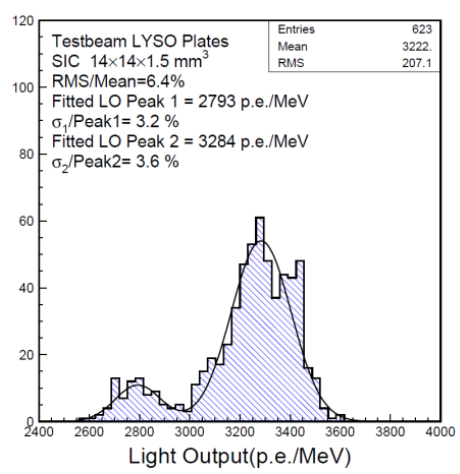

Figure 15. LO of 623 LYSO plates for a Shashlik matrix

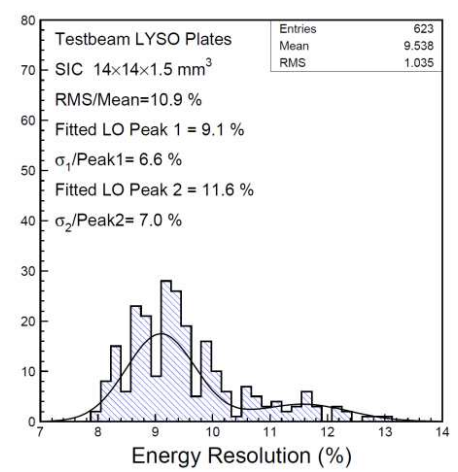

Figure 16. ER of $623 \mathrm{LYSO}$ plates for a Shashlik matrix

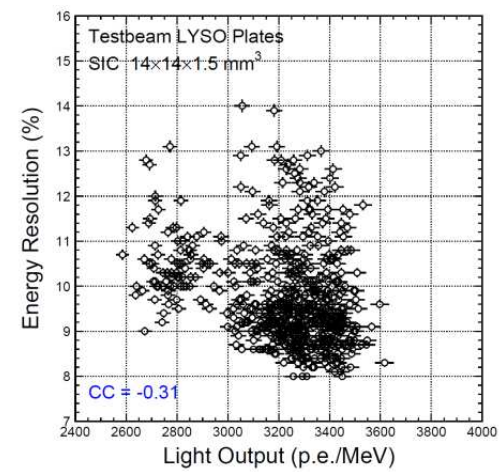

Figure 17. Correlation between the LO and the ER of 623 plates

Figure 18 shows very consistent transmittance at $420 \mathrm{~nm}$ for all LYSO plates except two with

125 significantly lower transmittance. Figure 19 shows no correlation between the LO and the 

effect on the LO.

129

130

\section{1}

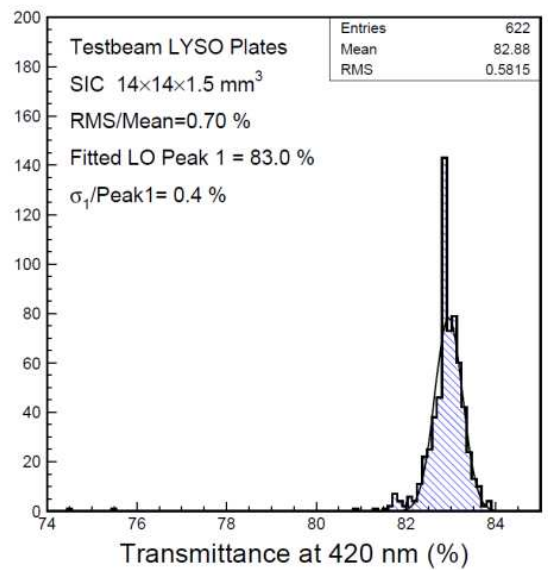

Figure 18. Transmittance at $420 \mathrm{~nm}$ is shown for LYSO plates foe a Shashlik matrix

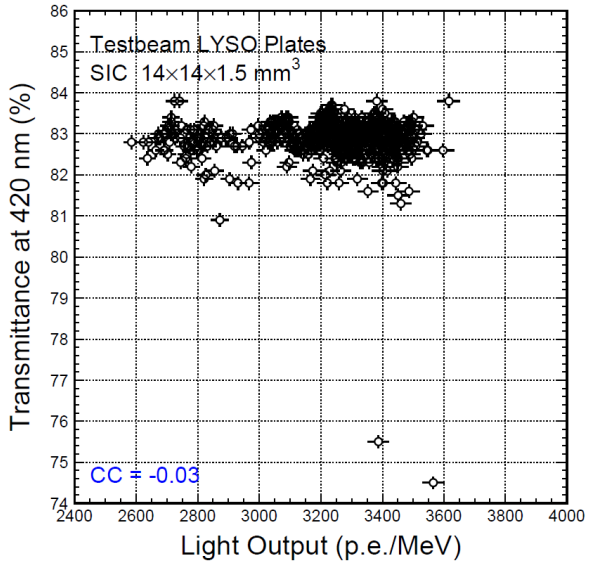

Figure 19. Correlation between the LO and the transmittance at $420 \mathrm{~nm}$ of 623 plates

\section{Summary}

Long LYSO crystals produced in industry show good transmittance exceeding $75 \%$ specification at $420 \mathrm{~nm}$ and good FWHM energy resolution better than $12.5 \%$ specification at $511 \mathrm{keV}$. Typical LO spread is at a level of $6 \%$ for long crystals, which may be reduced to about $3 \%$ in mass production. Correlations are observed between the LO and the LT at $420 \mathrm{~nm}$ as well as between LO and decay time for long LYSO crystals. A slight anti-correlation was observed between the LO and the energy resolution for $14 \times 14 \times 1.5 \mathrm{~mm}$ LYSO plates. Results of these investigations indicate that the quality of LYSO crystals grown in industry is adequate for future HEP calorimeters at both the energy and intensity frontiers.

\section{Acknowledgments}

This work was supported in part by the US Department of Energy Grant DE-SC0011925. 
144 References

145 [1] Melcher C L and Schweitzer J S, 1992, IEEE T Nucl Sci, 39, 502.

146 [2] Melcher C L and Schweitzer J S, 1992, Nucl Instrum Meth A, 314, 212.

147 [3] Kobayashi M, Ishii M, and Melcher C L, 1993, Nucl Instrum Meth A, 335, 509.

148 [4] Daghighian F, Shenderov P, Pentlow K S, Graham M C, Eshaghian B, Melcher C L, et al., 149 1993, IEEE T Nucl Sci, 40, 1045.

150 [5] Ren G H, Wang S H, Li H Y, and Lu S, 2003, J Inorg Mater, 18, 269.

151 [6] Kimble T, Chou M, and Chai B H T, 2002, Proc. IEEE Nuclear Science Symp. Conf., vol 3 152 (Norfolk, Virginia, USA), p 1434.

153 [7] Cooke D W, McClellan K J, Bennett B L, Roper J M, Whittaker M T, Muenchausen R E, et 154 al., 2000, J Appl Phys, 88, 7360.

155 [8] Vilardi I, Braem A, Chesi E, Ciocia F, Colonna N, Corsi F, et al., 2006, Nucl Instrum Meth A, $156 \quad \mathbf{5 6 4}, 506$.

157 [9] Chen J M, Mao R H, Zhang L Y, and Zhu R Y, 2007, IEEE T Nucl Sci, 54, 718.

158 [10] Chen J M, Zhang L Y, and Zhu R Y, 2005, IEEE T Nucl Sci, 52, 3133.

159 [11] Zhang L Y, Mao R H, Yang F, and Zhu R Y, 2014, IEEE T Nucl Sci, 61, 483.

160 [12] Eigen G, Zhou Z, Chao D, Cheng C H, Echenard B, Flood K T, et al., 2013, Nucl Instrum $161 \quad$ Meth A, 718, 107.

162 [13] SuperB Conceptual Design Report, INFN/AE-07/2, SLAC-R-856, LAL 07-15, Mar. 2007

163 [14] Chen J M, Mao R H, Zhang L Y, and Zhu R Y, 2007, IEEE T Nucl Sci, 54, 1319.

164 [15] Mao R H, Zhang L Y, and Zhu R Y, 2012, IEEE T Nucl Sci, 59, 2224.

165 [16] Zhang L Y, Mao R H, and Zhu R-Y, 2009, IEEE Nuclear Science Symp. Conf. (Orlando, $166 \quad$ Florida, USA), p 2041.

167 [17] Dissertori G, Luckey D, Nessi-Tedaldi F, Pauss F, Quittnat M, Wallny R, et al., 2014, Nucl $168 \quad$ Instrum Meth A, 745, 1.

169 [18] Mao R H, Zhang L Y, and Zhu R Y, 2008, IEEE T Nucl Sci, 55, 1759. 